\title{
Tiempo de sangría de IVY modificado: Valores normales en edad pediátrica
}

\author{
Dr. Ërnesto Ríos L., TM Mónica Pinochet C. y TM Mónica Mandujanó
}

Modified Ivy's bleeding time; normal values in children.

\begin{abstract}
The bleeding time (template) is studied in 110 pediatric patients. The normal values obtained are $\vec{x}: 5^{\prime} 45^{\prime \prime} \pm 60^{\prime \prime}$ ( $1 \mathrm{SD}$ ) and the range of the $95 \%$ confidence is $3^{\prime} 45^{\prime \prime}$ to 7 ' $45^{\prime \prime}$. There are not differences of bleeding time in the pediatric ages. The maximal difference between the bleeding time of the duplicate is 1' $30^{\prime \prime}$.

A comment of the bleeding time technics is done remarking the benefits of reproducibility and sensibility of the template technic.

The normal vahes of platelets counts $\times \mathrm{mm}^{3}$ is $\bar{x} 275.000 \pm 60.000$ (1 SD) with a range of $95 \%$ confidence of 150.000 to 400.000 .
\end{abstract}

El tiempo de sangría es un examen basico de orientación en el estudio de la hemostasis de un paciente. Es un método "in vivo", que en condiciones fisiológicas mide la capacidad de las plaquetas para funcionar normalmente formando el "tapón plaquetario" de la hemostasis primaria. Para la interpretación de la función plaquetaria normal o patológica se requiere que el recuento de plaquetas sea superior a $100.000 \mathrm{x} \mathrm{mm}^{3} 1$

El tiempo de sangría es un examen sencillo, de gran uso y utilidad, por ello es necesario determinar el rango de sus valores normales. De esta manera se define tambien el nivel patológico que dará una orientación clínica importante en el preoperatorio o en el enfermo que sangra.

Fue nuestro interés determinar el tiempo de sangría en las diferentes edades pediátricas, usando un método bien standarizado y obtener sus rangos normales de variación.

\section{MATERIAL Y METODOS}

Se estudiaron 110 niños de ambos sexos de 3 meses a 18 años de edad. En todos ellos se efectuó una historia orientada, para descartar antecedentes clínicos de sangramiento personal o familiar. Se exigió además no haber ingerido ningún medicamento en los 10 días previos, haciendo especial mención del ácido acetilsalicílico.

Todos los niños fueron estudiados en una etapa

\footnotetext{
1 Unidad de Hematologia-Hospital L. Calvo Mackenna
}

previa a ser intervenidos quirúrgicamente. El tiempo de sangría constituyó un examen más del preoperatorio. Las patologias quinúrgicas no fueron de urgencia y en su gran mayoría estuvieron constituidas por hernias. El estaco general y nutritivo de los niños fue adecuado.

Se usó el tiempo de sangría de Ivy modificado (template) en duplicado ${ }^{2}$. El examen consiste en efectuar una incisión standarizada en el antebrazo, mientras se mantiene una presión de $40 \mathrm{~mm}$ de mercurio en el brazo, usando un esfingomanómetro corriente. En el antebrazo se ubica una zona sin vasos superficiales, efectuándose una incisión de $1 \mathrm{~cm}$ de longitud y $1 \mathrm{~mm}$ de profundidad. Para este efecto se usó un aparato diseñado en la Universidad de Chile por los Drs. Uribe y Honorato. Figura 1 y 2 . El tiempo de sangría es el lapso

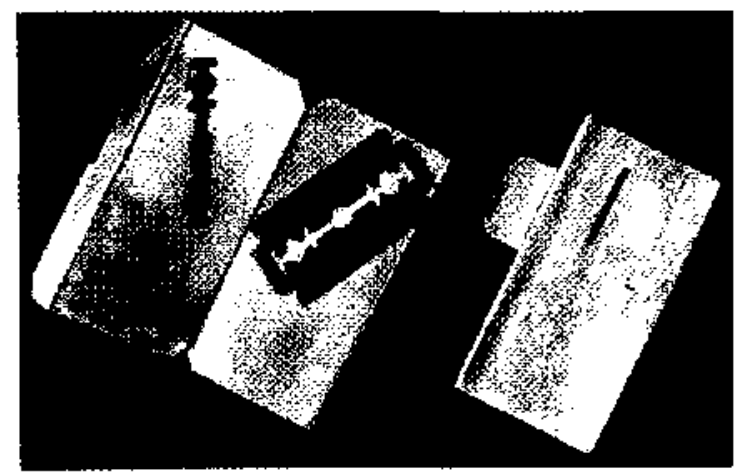

Figura 1.

El aparato para efectuar el tiempo de sangría de Ivy modificado se muestra con sus dos partes. El elemento cortante es una hoja de afeitar corriente. 


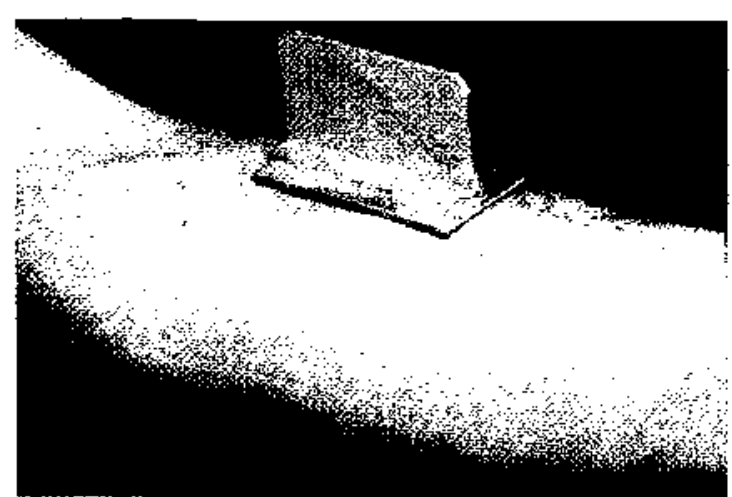

Figura 2.

El aparato ubicado en el antebrazo listo para efectuar ta incisión de $1 \mathrm{~cm}$ de largo y $1 \mathrm{~mm}$ de profundidad.

que media entre la incisión y el término del sangramiento espontáneo. Cada medio minuto se absorve el exceso de sangre con papel filtro, que no debe tocar los bordes de la herida.

En cada niño se efectuó además un recuento de plaquetas en duplicado. Se usó la vía capilar y el microscopio de fase contrastada según el método de Brecher y Cronkite ${ }^{3}$. Los niños que tuvieron un recuento plaquetario $<150.000 \times \mathrm{mm}^{3}$ fueron descartados del estudio.

Se efectuó un análisis estadístico usando el test de varianza y el test $T$ student.

\section{RESULTADOS}

Los pacientes estudiados fueron divididos arritrariamente en 18 grupos a saber: lactantes de 3 a 12 meses y de 1 a 2 años. Preescolares de 2 a 3 años, de 3 a 4 años, de 4 a 5 años y de 5 a 6 años. Escolares de 6 a 7 años, de 7 a 8 años, de 8 a 9 affos de 9 a 10 anos, de 10 a 11 años y 11 a 12 años. Los adolescentes fueron divididos según edad $y$ sexo en 6 grupos de 12 a 14 años, de 14 a 16 años y de 16 a 18 años.

Los resultados de los tiempos de sangría (promedio del duplicado) de estos 18 grupos fueron estudiados con un análisis de varianza, no observándose diferencias significativas. $(\mathrm{F}=1.18$ p> 0.05).

Con estos resultados los pacientes se reagruparon en 4 categorías lactantes, preescolares, escolares y adolescentes, cuyos valores se observan en la tabla 1. Un nuevo análisis de varianza de estos 4

\section{Tabla 1.}

Tiempo de Sangría (Ivy modificado) y recuen to de plaquetas en diferentes grupos pediátricos.

\begin{tabular}{|c|c|c|c|}
\hline Gaupo Etario & No & T. Sargría $(x \pm I D S)$ & Rcto. Plaquetas ( $\bar{x} \pm 1$ DS) \\
\hline Lactertes & 19 & $6.06^{\prime \prime} \pm 62^{\prime \prime}$ & $242.250 \pm 47.000$ \\
\hline Preesoolarss & 30 & $5^{\prime} 33^{\prime \prime} \pm 43^{\prime \prime}$ & $266.724 \pm 47.000$ \\
\hline Escolanes & 30 & $S^{\prime} 29^{\prime \prime} \pm 64^{\prime \prime}$ & $311.550 \pm 79.000$ \\
\hline Adolescentea & 31 & 5 51" $564 "$ & $275.892 \pm 48.000$ \\
\hline To1日l & 110 & $5^{\prime} 44^{\prime \prime} \pm 60^{\prime \prime}$ & $275.673 \pm 63.000$ \\
\hline
\end{tabular}

grupos tampoco reveló diferencias significativas en los resultados $(F=2.46 \mathrm{p}>0.05)$. De esta manera se concluye que no existen diferencias entre distintas edades y que el tiempo de sangría de lvy modificado normal en 110 pacientes pediátricos es $\bar{x} 5^{\prime} 44 " \pm 60 "$ (1 DS) (Fig. 3) siendo el rango de

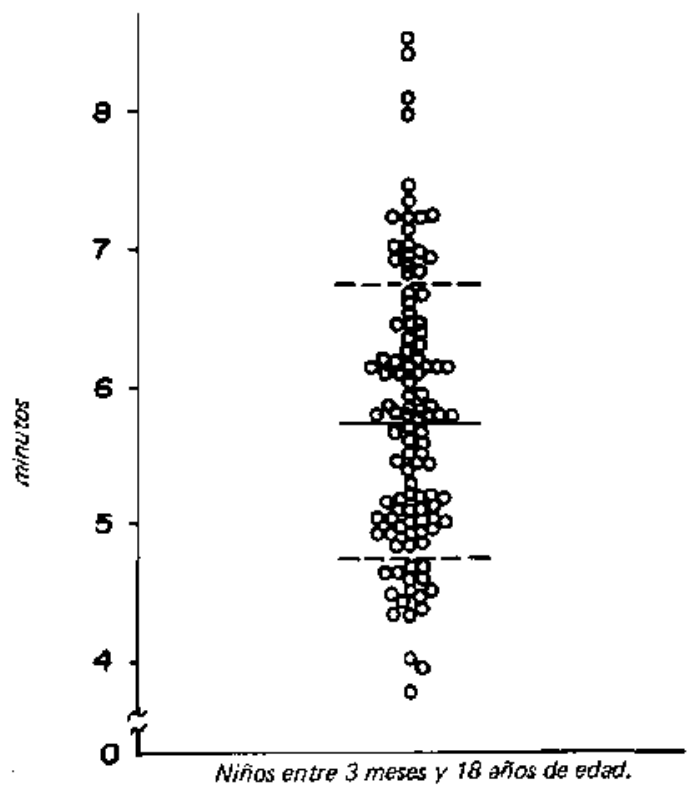

Figura 3.

Valores del tiempo de sangría de lvy modificado en 110 niños normales. Se observa el promedio y I desviación standard.

normalidad del $95 \%$ de confianza entre 3' 44 " y 7' 44".

El recuento normal de plaquetas $\mathrm{x} \mathrm{mm}^{3}$ reveló $\bar{x} 275.673 \pm 63.000$ siendo el rango de normalidad del $95 \%$ de confianza de $149.673 \mathrm{x} \mathrm{m}^{3}$ a $401.673 \times \mathrm{mm}^{3}$.

\section{DISCUSION}

El tiempo de sangria es un examen de orientación en el estudio de la hemostasis de un paciente, ha sido largamente usado desde su incorporación a comienzos de siglo. Se han usado diferentes técnicas que han ido mejorando el rendimiento del examen.

La primera técnica fue descrita por $D_{u k e}^{4}$ en 1910 y consiste en una punción en el lóbulo de la oreja y la medición del tiempo hasta que cese el sangramiento espontâneo. Esta ténica se ha demostrado poco reproducible de un paciente a otro y poco sensible, detectando sólo los casos extremos de patologia 5 .

En 1941 Ivy $^{6}$ describe otra técnica más estandarizada que la anterior. En ella se coloca un manguito en el brazo para ejercer una presión de $40 \mathrm{~mm}^{3}$ de mercurio en el territorio vascular 
distal, similar en todos los enfermos. En el antebrazo se efectúan 3 punciones en las cuales se mide el tiempo de sangria.

En 1958 Borchgrevink ${ }^{7}$ reemplaza las punciones del antebrazo por incisiones y finalmente en 1969 Mielke $^{2}$ describe el método de Ivy modificado o template en que la técnica tiene una máxima estandarización. En todos los enfermos la presión ejercida sobre el territorio vascular es $40 \mathrm{~mm}$ y en todos ellos la incisión es de una longitud y profundidad igual.

El análisis del tiempo de sangría demostró que no existen variaciones en pacientes de diferentes edades. Este hecho es importante, ya que últimamente se ha señalado que existen varios parámetros hematológicos que tienen cambios con el desarrollo ${ }^{8}$. Tampoco hubo diferencias con los valores del adulto.

En el presente estudio el tiempo de sangría fue efectuado en duplicado. Se analjzó además cada uno de los resultados de un mismo paciente por separado.

Las diferencias obtenidas entre el 1.er tiempo de sangría y el 2.0 fueron analizadas con un test $T$ para muesiras pareadas. Se mostró que no existió error de técnica ya que no hubo diferencias significativas $(t=1.19 \mathrm{p}>0.20)$. Se analizó además cual era la diferencia máxima debida al azar entre los 2 tiempos de sangria del dupticado; por existir una distribución asimétrica de las diferencias se usó el método de la mediana y percentjles. El percentil 95 reveló un valor de 91 segundos. Diferencias mayores en el duplicado indican un problema de técnica.

\section{RESUMEN}

Se estudió el tiempo de sangría de lvy modificado en 110 pacientes pediátricos. Los valores normales mostraron = : 5 ' $45^{\prime}$ " y 1 DS: 60 ", siendo el rango del $95 \%$ de confidencia de 3 ' $45^{\prime \prime}$ a
7' 45'. No se demostró diferencias del tiempo de sangría en las distintas edades pediátricas. La diferencia máxima entre 2 tiempos de sangría del duplicado es 1' 30 '.

Se comenta sobre las diferentes técnicas del tiempo de sangría, siendo la usada en este estudio la más reproducible y sensible por su mayor nivel de standarización.

Los valores normales del recuento de plaquetas

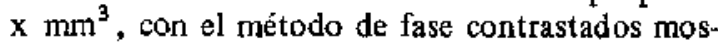
traron $=: 275.000$ y 1 DS 60.000 siendo el rango del $95 \%$ de confidencia 150.000 a 400.000 .

\section{REFERENCIAS}

${ }^{1}$ L.A. Harker and S.J. Stichter: The bleeding time as a screening test for evaluation of platelet.function. $N$. Engl. J. Med. 287: 155, 1972.

${ }^{2}$ C.H. Mielke, M.M. Kaneshoro, I.A. Maher, J.M. Weiner and S.I. Rapeport: The standardized normal Ivy bleeding time and its prolongation by aspirin. Blood 34 : $204,1969$.

${ }^{3} G$. Brecher, M. Schneiderman and E.P. Cronkite: The reproducibility and constancy of the platelet count. Amer. J. Clin. Path. 23: 15, 1953.

4 W.W. Duke: The relation of blood platelets to hemorrhagic disease: description of a method for determining the bleeding time and coagulation time and report of three cases of hemorrhagic discase relieved by transfusion JAMA 55: $1185,1910$.

5 F. Bachmann: Diagnostic approach to mild bleeding disorders. Seminars in Hematology 17: 292, 1980.

- A.C. Ivy, D. Netson and G. Bucher: The standarization of certain factors in the eutaneous "venostasis" bleeding time technique. J. Lab. Clin. Med. 26: 1812, 1941.

${ }^{7}$ C.F. Bochgrevink and B.A. Waaler: The secondary bleeding tine: a new method for the differentiation of hemorrhagic deseases. Acta Med. Scand. 162: 361, 1958.

${ }^{8}$ E. Rios, $M$. Olivares y A, Stekel: Nutrición de hierro en lactantes: definición de valores normales y prevalencia de la carencia de híerro. XVI Congreso Sociedad Latinoamericana de Investigaciones Pediátricas (SLAIP) Viña del Mar, Chile 1978. 